\title{
Improving But-For Delay Analysis and Concurrency Assessment
}

\author{
Moneer Bhih ${ }^{1}$ and Tarek Hegazy ${ }^{2}$
}

1 University of Waterloo, Canada, Formerly, Univ. of Benghazi, mbhih@uwaterloo.ca

2 University of Waterloo, Waterloo, Canada, terek@uwaterloo.ca

\begin{abstract}
But-For analysis is one of the popular techniques for apportioning the responsibility for project delays among the project parties (owner, contractor, and third party). Despite its acceptance by courts, one of its known drawbacks is that it produces conflicting results when adopting different party's viewpoints. Moreover, But-For analysis is not able to identify the concurrent delays caused by multiple parties. Despite some literature modifications to address those shortcomings, Modified But-For (MBF) analysis persistently does not consider event chronology and thus can produce wrong results. This paper thus discusses the concurrency assessment method of the MBF and introduces implementation improvements to divide the analysis into multiple windows to increase the analysis resolution, account for critical path fluctuations, and consider the chronology of different-party events, which is a requirement by recent delay analysis guidelines of professional bodies such as AACE and ASCE. A case study is used to show a detailed procedure for applying multiple-window MBF analysis to produce more accurate and repeatable delay analysis, considering concurrent delays.
\end{abstract}

(c) 2020 The Authors. Published by Budapest University of Technology and Economics \& Diamond Congress Ltd Peer-review under responsibility of the Scientific Committee of the Creative Construction Conference 2020.

Keywords: but-for, collapsed as-built, concurrent delay, construction, delay analysis

\section{Introduction}

The risky and uncertain nature of construction projects frequently leads to schedule delays, cost overruns, and disputes [1]. Delay analysis techniques are the main forensic tools to fairly apportion construction delays, settle disputes, and avoid its undesirable effects such as lawsuits and contract termination [2]. Among several existing delay-analysis methods, the But-For (or collapsed as-built [3]) technique is one of the most widely used and accepted techniques $[4,5,6]$ because of its simplicity and its use of the as-built schedule (the real schedule already executed) $[7,8]$. However, the traditional But-For method exhibit four main drawbacks: (1) it produces different results when adapting different parties' viewpoints [9, 10, 11]. This shortcoming made the technique easy to manipulate [12] to support different parties' claim argument [13]; (2) it is unable to identify the concurrent delays that occurred during execution[14, 15]; (3) it is insensitive to the specific timing of parties' events on the schedule; and (4) it is insensitive to the fluctuation of the critical path(s) during construction. In the literature, the common misinterpretation of results in the traditional But-For were explained and resolved by Bhih and Hegazy [9]. Also, Mbabazi et al. [16] introduced the Modified But-For (MBF) method to address the first two drawbacks, thus producing consistent results irrespective of the adopted viewpoint, and accurately identifying concurrent delays. Despite the improvements introduced by the MBF, the two latter drawbacks ( 3 and 4 ) still persist due to the fact that MBF uses a single time window (the whole as-built schedule) for the analysis [17]. To support schedule forensic analysis, several professional bodies have established general industry guides. Currently, three schedule-analysis guidance documents are available [18]: the Delay and Disruption Protocol of the Society of Construction Law (SCL) in the UK [19]; the Forensic Schedule Analysis Recommended Practice $29 \mathrm{R}-03$ of 
the AACEI in the USA [20]; and the Schedule Delay Analysis (ANSI/ASCE/CI 67-17) of the ASCE in the USA [21]. These guides, however, provide generic guidelines to promote balanced and fair assessment of compensations and time extensions rather than specific steps to perform delay analysis.

This paper, first discusses the MBF method and how it improves concurrency assessment of the traditional But-For method. Afterwards, the paper introduces improvements to the MBF to address the insensitivity to event chronology and critical path fluctuations. Example applications are used to discuss and validate the developments made.

\section{Modified But-For method}

The MBF methodology [16] allocates project delays in the general case of three parties (owner, contractor, and third party). In this paper, for simplicity, the case of two parties is used. MBF was introduced to resolve the drawbacks of the traditional But-For of producing different results when different parties' viewpoints are adopted [11, 16, 22] and its inability to identify concurrent delays [9]. A small case study of four activities (adopted from literature [23]) is used to explain MBF benefits. The as-planned and as-built schedules are shown in Fig. 1. The project was planned to finish in 10 days (Fig. 1a). The as-built schedule (Fig. 1b) exhibits simultaneous owner and contractor delays so the project finished 2 days behind schedule. The owner event to activity $B$ shifted the remaining portions of activities B and C to a period of lower productivity, thus taking longer time than planned and delaying the project.
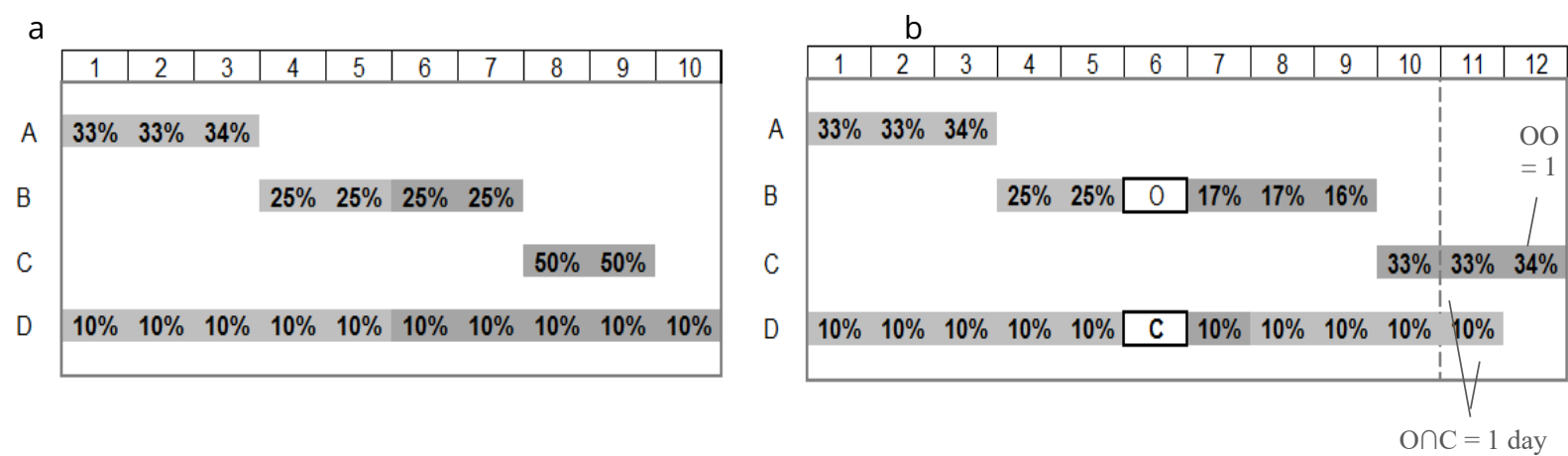

Fig. 1. Case study schedules (a) As-planned schedule, (b) As-built Schedule (Scenario 1)

In traditional But-For analysis, parties' events are removed one at a time from the as-built schedule to investigate its impact on the project duration. From the owner's perspective, by removing the owner-caused events from the as-built schedule it collapses 1 day; so the owner accepts responsibility for only 1 day project delay, and the remaining balance of project delays (1 day) is attributed to the contractor. Thus, Owner's viewpoint results are (Owner responsibility $=1$ day, Conctractor responsibility $=1$ day). From the contractor's perspective, on the other hand, since the as-built schedule does not change after removing the contractor events, then the contractor's responsability is 0 days and the remaining balance ( 2 days) is attributed to owner. Thus, Contractor's viewpoint results in (Owner responsibility $=2$ day, Contractor responsibility $=0$ days). As such, the difference in results and the inability to identify concurrent delays have become serious drawbacks to the traditional But-For method.

To resolve these issues, the MBF method [16] utilizes Venn representation and set theorem to allocate delay responsibility among project parties, as shown in left side of Fig. 2 . The two intersecting circles represent the project delays caused by Only-Owner (OO), Only-Contractor (OC), and the intersection area of their Concurrent Delays $(O \cap C)$, as shaded in the figure. Using set theory, a formulation of all segments in the Venn representation can be made to calculate the three areas of delay responsibilities, which add up to the total project delay, as follows: 


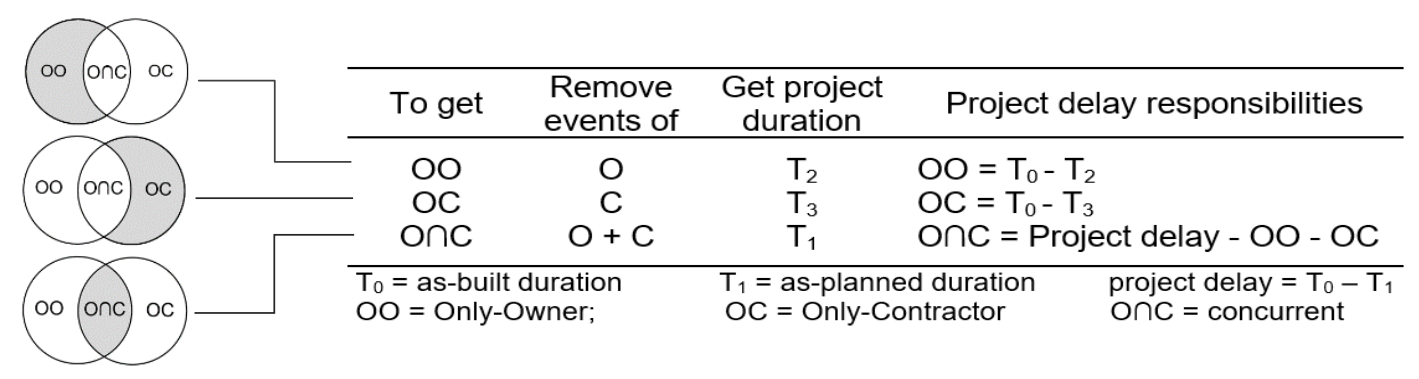

Fig. 2. MBF methodology

Applying the MBF method to the case in Fig. 1 is shown in the steps of Fig. 2 . The MBF process basically removes three combinations of delay events from the as-built schedule, one at a time, and the resulting project duration is used in the delay responsibility calculations. Having the as-built duration of $T_{0}=12$, the first MBF step is to remove all the Owner $(\mathrm{O})$ events from the as-built schedule. Accordingly, without any owner events, the schedule becomes $T_{2}=11$ days. Therefore, the Only-Owner (OO) responsibility is directly evaluated as the difference between $\mathrm{T}_{0}$ and $\mathrm{T} 2$, i.e., $\mathrm{OO}=\mathrm{T}_{0}-\mathrm{T}_{2}=12-11=1$ day. In the second step, removing all the Contractor events from the as-built, the schedule duration becomes $T_{3}=12$ days, thus, the OnlyContractor $(\mathrm{OC})$ responsibility $=\mathrm{T}_{0}-\mathrm{T}_{3}=12-12=0$ days. Also, in the third step, removing both the owner and contractor events results in the as-planned duration $T_{1}=10$ days. Accordingly, using Equation 1, concurrent delays $O \cap C=$ Project delays $-O O-O C=\left(T_{0}-T_{1}\right)-O O-O C=1$ day. Summary results are shown in Table 1 ( $O O=1$ day, $O C=0, O \cap C=1$ day) and these values can be easily observed on the as-built schedule of Fig. $1 \mathrm{~b}$. This analysis, as such, is repeatable and does not depend on any party's viewpoint. Furthermore, it does not ignore concurrent delays.

Table 1. Case study MBF calculations (scenario 1)

\begin{tabular}{cccl}
\hline To get & $\begin{array}{c}\text { Remove } \\
\text { events }\end{array}$ & $\begin{array}{c}\text { Project duration } \\
\text { (days) }\end{array}$ & Project delay responsibilities (days) \\
\hline $\mathrm{OO}$ & $\mathrm{O}$ & $\mathrm{T}_{2}=11$ & $\mathrm{OO}=\mathrm{T}_{0}-\mathrm{T}_{2}=12-11=1$ \\
$\mathrm{OC}$ & $\mathrm{C}$ & $\mathrm{T}_{3}=12$ & $\mathrm{OC}=\mathrm{T}_{0}-\mathrm{T}_{3}=12-12=0$ \\
$\mathrm{O} \cap \mathrm{C}$ & $\mathrm{O}+\mathrm{C}$ & $\mathrm{T}_{1}=10$ & $\mathrm{O} \cap \mathrm{C}=$ Project delays $-\mathrm{OO}-\mathrm{OC}=\mathrm{T}_{0}-\mathrm{T}_{1}-\mathrm{OO}-\mathrm{OC}=1$ \\
\hline $\mathrm{T}_{0}=12$ & & &
\end{tabular}

\section{Improving MBF sensitivity to event chronology}

As the MBF method uses the entire project as a single analysis-window, by nature, it cannot respect events' chronology, i.e., the specific timing of the events. Furthermore, the MBF only considers the final critical path(s) of the as-built schedule, overlooking path fluctuations over the course of the project, which affect the analysis results. To highlight this issue, the as-built schedule of the case study is slightly altered as shown in Fig. 3. In this scenario, the contractor and owner events were shifted so that each one is happening on different day, thus are no longer simultaneous.

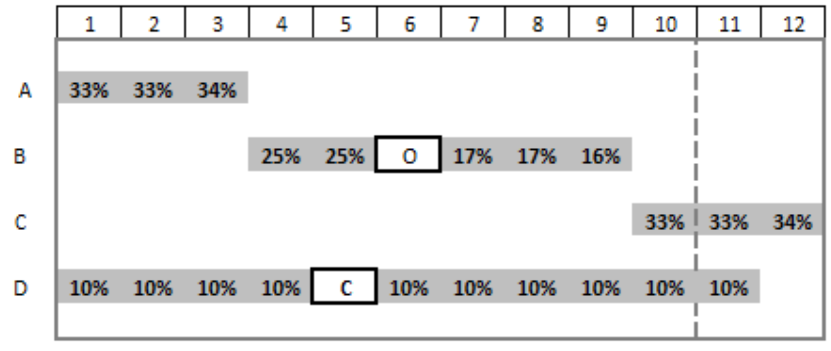

Fig. 3. Case study as-built Schedule (Scenario 2)

Using a single-window MBF, scenario 2 was analysed by following the step-by-step process explained earlier. The results are shown in Table 2 being $(\mathrm{OO}=1$ day, $\mathrm{O} \cap \mathrm{C}=1$ day). These results are identical to those 
obtained for the original case study (scenario 1) in spite of the difference in the timing of events. This shows that the MBF analysis is not sensitive to the timing of delay events.

Table 2. Case study MBF calculations (scenario 2)

\begin{tabular}{cccl}
\hline To get & $\begin{array}{c}\text { Remove } \\
\text { events }\end{array}$ & $\begin{array}{c}\text { Project duration } \\
\text { (days) }\end{array}$ & Project delay responsibilities (days) \\
\hline $\mathrm{OO}$ & $\mathrm{O}$ & $\mathrm{T}_{2}=11$ & $\mathrm{OO}=\mathrm{T}_{0}-\mathrm{T}_{2}=12-11=1$ \\
$\mathrm{OC}$ & $\mathrm{C}$ & $\mathrm{T}_{3}=12$ & $\mathrm{OC}=\mathrm{T}_{0}-\mathrm{T}_{3}=12-12=0$ \\
$\mathrm{O} \cap \mathrm{C}$ & $\mathrm{O}+\mathrm{C}$ & $\mathrm{T}_{1}=10$ & $\mathrm{O} \cap \mathrm{C}=$ project delays $-\mathrm{OO}-\mathrm{OC}=2-1-0=1$ \\
\hline $\mathrm{T}_{0}=12$ & & &
\end{tabular}

Irrespective of the identical results of the two scenarios, they represent two cases of concurrency, namely "true concurrency" versus "concurrent effect", as defined by the SCL protocol [19]. This protocol defines concurrent effects as "two or more delay events arise at different times, but the effects of them are felt at the same time". As shown in the above analysis, single-window techniques such as MBF suffers from its inability to differentiate between true concurrent delays (as in scenario 1) and concurrent effects (as in scenario 2) due to its inability to consider event chronology. Because this drawback can seriously affect delay analysis results, the ASCE proposed standard [21] and the AACEI recommended practice [20] require that project delays be assessed chronologically and cumulatively.

\subsection{Analysis considering event chronology}

To correctly analyze the project delays of scenario 2, the chronological order of events is considered. Starting from the as-planned schedule of Fig. 1a, the schedule has one critical path (task D) and one nearcritical path (A-B-C) with 1 day of float. As shown in Fig. 4, the contractor event on day 5 caused 1-day project delay, resulting in an expected project completion on day 11, and this also increased the float of the nearcritical path (A-B-C) to 2 days. Afterwards, on day 6 , the owner event on path (A-B-C) not only consumed all the float but also caused an additional project delay, ending in day 12. Thus, the correct responsibility for scenario 2 is $(O O=1$ day, $O C=1$ day, $O \cap C=0$ days). Such logical analysis cannot be done manually for large and complex projects, and modifications to the MBF are needed.

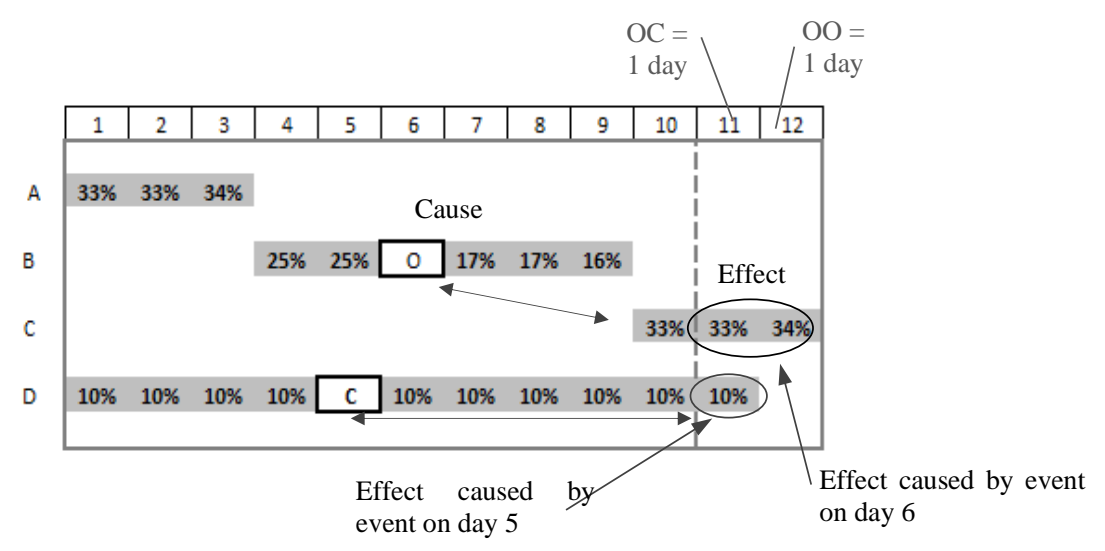

Fig. 4. Analysis based on event chronology

\subsection{MBF with multiple-window analysis}

To overcome the MBF insensitivity to the timing of events and facilitate accurate concurrency assessment, this paper proposes to use multi-window MBF analysis. Multi-window MBF analysis will also improve the tracking of critical path(s) fluctuations. The analysis windows should be selected carefully as the window size could affect the results. Since the MBF method can assess project delays based only on effects regardless of its causing events, having different parties' events in separate analysis windows will ensure that their effects will be assessed separately, unless the events happened simultaneously or very close in time. For instance, in scenario 2, if a literal concurrency is adopted (i.e., concurrent events must ha happen 
on same day), using two analysis windows (Window 1: days 1 to 5; and Window 2: days 6 to 12), as shown in Fig. 5, will ensure arriving at the correct answer.

$a$

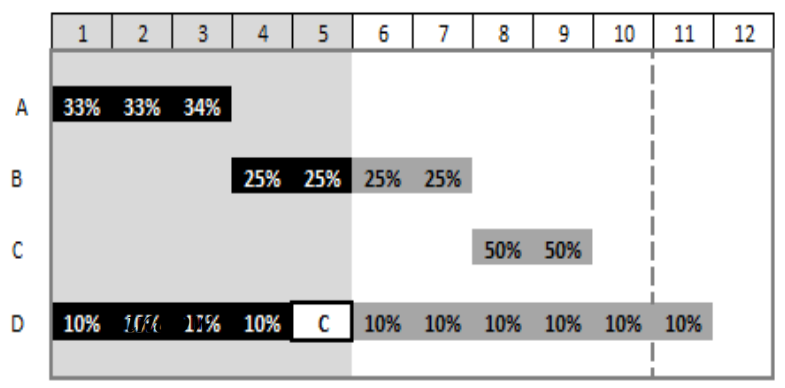

$b$

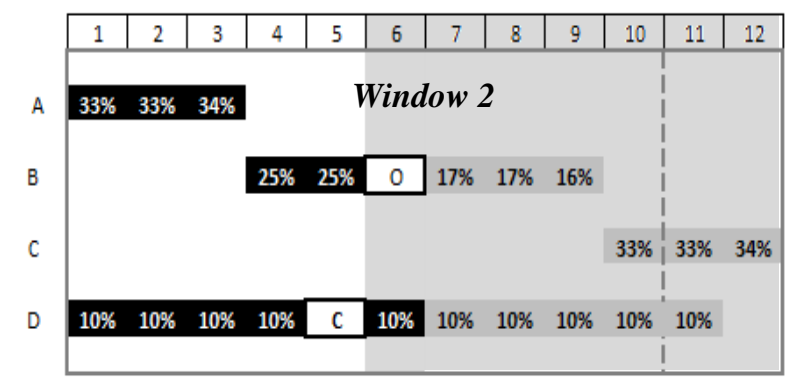

Fig. 5. Analysis of scenario 2 (a) Window 1, (b) Window 2

Applying the two-window MBF analysis to scenario 2 is shown in Table 3. Each window is analysed separately and the result is shown in the corresponding row; finally the analysis results are accumulated to obtain the project delay responsibility. The results obtained using this multi-window analysis are identical to the correct logical results obtained considering event chronology and those obtained by Zhang \& Hegazy [23] using the more accurate daily-windows analysis. As such, applying multi-window MBF analysis can produce accurate results by properly separating the different parties' events in different windows. This in fact increases the analysis resolution and makes the MBF analysis capable of better tracking of the critical path(s) fluctuations and the chronology of delay events.

Table 3. Case study MBF multi-window calculations (scenario 2)

\begin{tabular}{ccccccccc}
\hline \multirow{2}{*}{ Window } & \multirow{2}{*}{ Days } & \multicolumn{2}{c}{ As-built without party(ies) events (days) } & \multicolumn{2}{c}{ Project delay responsibilities (days) } \\
\cline { 3 - 9 } & & As-built & $\mathrm{O}$ and C & $\mathrm{O}$ & $\mathrm{C}$ & $\mathrm{OO}$ & $\mathrm{OC}$ & $\mathrm{O} \cap \mathrm{C}$ \\
\hline 1 & $1-5$ & $\mathrm{~T}_{0}$ & $\mathrm{~T}_{1}$ & $\mathrm{~T}_{2}$ & $\mathrm{~T}_{3}$ & $\mathrm{O}$ & 1 & 0 \\
2 & $6-12$ & 12 & 11 & 10 & 10 & 0 & 0 & 0 \\
\hline
\end{tabular}

\section{Concluding remarks}

Traditional but-For technique has been criticized for its highly subjective nature and its inability to assess concurrent delays. The Modified But-For method, therefore, uses Venn representation to overcome those drawbacks by reconciling the opposing viewpoints of project parties, and having the ability to apportion concurrent delays. However, the MBF method, due to its single-window implementation, suffers from its inability to respect the chronology of delay events and to capture the critical path(s) dynamics. In this paper, the MBF methodology was used to arrive at repeatable independent results that compiles the view points of all parties and identify concurrent delays. Furthermore, a multiple-window MBF analysis is proposed to improve the analysis resolution and produce more accurate results considering events chronology and critical path fluctuations. A simple case study is presented to demonstrate the improvements made in multiple-window MBF analysis.

\section{References}

[1] F. Arif and A. Morad, "Concurrent delays in construction: International Legal Perspective," ASCE-Journal Leg. Aff. Disput. Resolut. Eng. Constr., vol. 6, no. 1, pp. 1-4, 2014, https://doi.org/10.1061/(ASCE)LA.1943-4170.0000134.

[2] W. Menesi, "Construction delay analysis under multiple baseline updates," University of Waterloo, 2007.

[3] J. Zack, "But-For Schedules - Analysis and Defense," AACE Int. Trans., pp. 1-6, 1999.

[4] B. J. Lifschitz, E. M. Barba, A. M. Lockshin, and R. R. Compa-, "A Critical Review of the AACEI Recommended Practice for Forensic Schedule Analysis," vol. 29, no. 4, pp. 1-10, 2009.

[5] W. S. Dale and R. M. D'Onofrio, Construction Schedule Delays, 2017 ed. THOMSON REUTERS, 2017.

[6] J. Zack, "Pacing delays-the practical effect," Cost Eng. (Morgantown, West Virginia), vol. 42(7), pp. 23-28, 2000. 
[7] D. Arditi and T. Pattanakitchamroon, "Selecting a delay analysis method in resolving construction claims," Int. J. Proj. Manag., vol. 24, pp. 145-155, 2006, https://doi.org/10.1016/j.jproman.2005.08.005.

[8] J.-B. Yang and P.-C. Yin, "Isolated Collapsed But-For Delay Analysis Methodology," J. Constr. Eng. Manag., vol. 135, no. 7, pp. 570578, 2009, https://doi.org/10.1061/(ASCE)CO.1943-7862.0000016

[9] M. Bhih and T. Hegazy, "Improving Concurrency Assessment and Resolving Misconceptions about the But-For Delay Analysis Technique," ASCE-Journal Leg. Aff. Disput. Resolut. Eng. Constr., vol. 12, no. 2, pp. 1-10, 2020, https://doi.org/10.1061/(ASCE)LA.1943-4170.0000378

[10] S. a. Fawzy and I. H. El-Adaway, "Contract Administration Guidelines for Effectively and Efficiently Applying Different Delay Analysis Techniques under World Bank Funded Projects," J. Leg. Aff. Disput. Resolut. Eng. Constr., vol. 5, no. February, p. 120915032631000, 2012, https://doi.org/10.1061/(ASCE)LA.1943-4170.0000104.

[11] N. Braimah, "Construction Delay Analysis Techniques-A Review of Application Issues and Improvement Needs," Buildings, vol. 3, no. 3, pp. 506-531, 2013, https://doi.org/10.3390/buildings3030506.

[12] M. Magdy and M. Georgy, "Delay Analysis Methodologies Used by Engineering and Construction Firms in Egypt," J. Leg. Aff. Disput. Resolut. Eng. Constr., vol. 11, no. 3, 2019, https://doi.org/10.1061/(ASCE)LA.1943-4170.0000293.

[13] G. R. Stumpf, "Schedule delay analysis," Cost Eng. (Morgantown, West Virginia), 2000.

[14] I. H. El-Adaway, S. a. Fawzy, R. Bingham, P. Clark, and T. Tidwell, "Different Delay Analysis Techniques Applied to the American Institute of Architects A201-2007 Standard Form of Contract," J. Leg. Aff. Disput. Resolut. Eng. Constr., vol. 6, no. 3, p. 02514001 , 2014, https://doi.org/10.1061/(ASCE)LA.1943-4170.0000145.

[15] SCL-DDP, Delay and Disruption Protocol, no. October. 2002.

[16] A. Mbabazi, T. Hegazy, and F. Saccomanno, "Modified But-For Method for Delay Analysis," J. Constr. Eng. Manag., vol. 131, no. 10, pp. 1142-1144, 2005, https://doi.org/10.1061/(ASCE)0733-9364(2005)131:10(1142).

[17] M. Bhih and T. Hegazy, "Concurrent Delays : Comparison among Forensic Analysis Recommended Practices," in CSCE 7th International Construction Conference/Construction Research Congress 2019, 2019, pp. CON117-1-10.

[18] J. Livengood, "Knowns and Unknowns of Concurrent Delay," vol. 9, no. 3, pp. 1-11, 2017, https://doi.org/10.1061/(ASCE)LA.19434170.0000224.

[19] SCL-DDP, Delay and Disruption Protocol, no. February. Society of Construction Law, 2017.

[20] AACE RP 29R-03, Forensic Schedule Analysis, no. 29. American Association of Cost Engineers, 2011.

[21] ASCE 67-17, Schedule delay analysis. 2017.

[22] W. S. Dale and R. M. D'Onofrio, "Reconciling Concurrency in Schedule Delay and Constructive Acceleration," Public Contract Law J., no. 39, pp. 161-230, 2010.

[23] K. Zhang and T. Hegazy, "Apportioning concurrent delays and accelerations using daily windows," in Construction Research Congress 2005: Broadening Perspectives - Proceedings of the Congress, 2005, no. 519, pp. 787-796.

[24] T. Hegazy and K. Zhang, "Daily Windows Delay Analysis," J. Constr. Eng. Manag., vol. 131, no. 5, pp. 505-512, 2005, https://10.1061/(ASCE)0733-9364(2005)131:5(505). 